\title{
The ACSL3-LPIAT1 signaling drives prostaglandin synthesis in non-small cell lung cancer
}

\author{
Maria Saliakoura ${ }^{1}$ Inés Reynoso-Moreno ${ }^{2}$ - Chiara Pozzato ${ }^{1}$ - Matteo Rossi Sebastiano ${ }^{1}$ - Mirco Galié ${ }^{3}$. \\ Jürg Gertsch ${ }^{2} \cdot$ Georgia Konstantinidou (1) ${ }^{1}$
}

Received: 25 September 2019 / Revised: 16 January 2020 / Accepted: 27 January 2020 / Published online: 7 February 2020

(c) The Author(s) 2020. This article is published with open access

\begin{abstract}
Enhanced prostaglandin production promotes the development and progression of cancer. Prostaglandins are generated from arachidonic acid (AA) by the action of cyclooxygenase (COX) isoenzymes. However, how cancer cells are able to maintain an elevated supply of AA for prostaglandin production remains unclear. Here, by using lung cancer cell lines and clinically relevant $\mathrm{Kras}^{\mathrm{G} 12 \mathrm{D}}$-driven mouse models, we show that the long-chain acyl-CoA synthetase (ACSL3) channels AA into phosphatidylinositols to provide the lysophosphatidylinositol-acyltransferase 1 (LPIAT1) with a pool of AA to sustain high prostaglandin synthesis. LPIAT1 knockdown suppresses proliferation and anchorageindependent growth of lung cancer cell lines, and hinders in vivo tumorigenesis. In primary human lung tumors, the expression of LPIAT1 is elevated compared with healthy tissue, and predicts poor patient survival. This study uncovers the ACSL3-LPIAT1 axis as a requirement for the sustained prostaglandin synthesis in lung cancer with potential therapeutic value.
\end{abstract}

\section{Introduction}

Arachidonic acid (AA) is a polyunsaturated fatty acid that, as arachidonate, is maintained at low concentrations, but it is highly abundant in its esterified form in membrane phospholipids. Therefore, the amount of AA is tightly controlled by the membrane phospholipid reacylation/deacylation cycle, known as the Lands cycle [1-3]. Depending on the cellular demand, AA can be released through phospholipid hydrolysis by phospholipase A2 (PLA2), phospholipase D, or phospholipase C pathways [4], and is then converted to

Supplementary information The online version of this article (https:// doi.org/10.1038/s41388-020-1196-5) contains supplementary material, which is available to authorized users.

Georgia Konstantinidou

georgia.konstantinidou@pki.unibe.ch

1 Institute of Pharmacology, University of Bern, 3010 Bern, Switzerland

2 Institute of Biochemistry and Molecular Medicine, University of Bern, 3012 Bern, Switzerland

3 Department of Neuroscience, Biomedicine and Movement, University of Verona, 37134 Verona, Italy prostaglandins by cyclooxygenases 1 and 2 (COX1 and COX2). Whereas COX1 is constitutively expressed, COX2 is induced by proinflammatory cytokines, and plays a central role in the insurgence of cancer inflammation and tumor progression [5, 6]. Notably, prostaglandins promote tumor growth both by directly activating signaling pathways, which control cancer cell proliferation, anchorageindependent growth, migration, and apoptosis, and by orchestrating interactions between tumor cells and the surrounding stromal cells, establishing an immunosuppressive tumor microenvironment [7-10].

Non-small cell lung cancer (NSCLC) constitutes about $85 \%$ of all lung malignancies, out of which $30 \%$ harbor KRAS mutations that are associated with aggressive, therapy-resistant tumors [11]. KRAS upregulates COX2, and the latter produces prostaglandins, including prostaglandin E2 (PGE2), in order to promote tumor growth and metastasis [12]. The transcriptional regulation of COX2 has been mainly attributed to MAPK signaling cascade, particularly to the ERK1/2, JNK/SAPK, and p38/RK/Mpk2 pathways [13-15]. However, it is unclear how KRAS is able to maintain a continuous supply of AA to feed COX2 and drive prostaglandin synthesis.

Mutant KRAS drives aberrant lipid metabolism in NSCLC by scavenging extracellular fatty acids [16, 17]. 
Indeed, we previously found that enhanced activity of the acyl-CoA synthetase long-chain 3 (ACSL3), an enzyme that catalyzes the activation of long-chain fatty acids to CoA thioesters, boosts extracellularly derived fatty acid activation in mutant KRAS NSCLC [17]. Knockdown of ACSL3 in NSCLC cell lines results in reduced cancer cell proliferation, while its deletion in mice suppresses Kras ${ }^{\mathrm{G} 12 \mathrm{D}}$ driven tumor initiation [17]. Thus, given the importance of ACSL3 in KRAS-driven tumorigenesis, and due to the fact that ACSL3 preferentially utilizes long-chain fatty acids, including arachidonate as substrates [18], we set out to investigate whether ACSL3 plays a role in mediating the KRAS-dependent prostaglandin production in lung cancer. To this aim, we combined mass spectrometry-based targeted lipidomics, in vivo cell-based assays, targeted genetic manipulations in cancer cells and in mouse models, as well as analysis of patient NSCLC samples.

\section{Results}

\section{ACSL3 promotes channeling of AA to phosphatidylinositols in NSCLC cells}

To understand whether ACSL3 is required to relay the KRAS-mediated AA cascade, we examined the lipid profile of the A549 NSCLC cell line upon ACSL3 knockdown by performing mass-spectrometry-based targeted lipidomics. We quantified the most abundant lipid species containing AA (20:4) in four phospholipid classes: phosphatidylcholine (PC), phosphatidylserine (PS), phosphatidylethanolamine (PE), and phosphatidylinositol (PI). We found that ACSL3 knockdown significantly altered the quantity of phospholipids containing AA in combination with 16:0 (palmitic), 16:1 (palmitoleate), 18:0 (stearate), and 18:1 (oleate) (Fig. 1a). Strikingly, the knockdown of ACSL3 resulted in a strong decrease in the AA content of PE and PI. Interestingly, we found a $40 \%$ decrease in PI containing C18:0-20:4 fatty acids (PI C18:0-20:4), the most abundant PI in mammalian cells [19-21] (Fig. 1a and Supplementary Fig. 1A). Of note, the knockdown of ACSL3 did not affect the protein levels of ACSL4, another ACSL with substrate preference for AA, underscoring the lack of compensatory effects (Supplementary Fig. 1B). These results strongly suggest that in mutant KRAS NSCLC cells, ACSL3 channels AA in glycerophospholipids.

PIs are reversibly phosphorylated at the inositol headgroup generating phosphoinositides, including phosphatidylinositol4 phosphate (PI4P) and the plasma membrane-localized phosphatidylinositol-4, 5 biphosphate, $\mathrm{PI}(4,5) \mathrm{P}_{2}$ [22, 23]. We hypothesized that a decrease in the most abundant PI, C18:0-20:4, would also affect the production of
C18:0-20:4 PI4P and C18:0-20:4 PI(4,5) $\mathrm{P}_{2}$. Indeed, upon ACSL3 knockdown, we observed a decrease in C38:4-PI4P and $\mathrm{C} 38: 4-\mathrm{PI}(4,5) \mathrm{P}_{2}$ lipids presumably due to the reduction of PI C18:0-C20:4 (Fig. 1b, c). This result additionally confirms a role of ACSL3 in esterifying AA into PI in mutant KRAS lung cancer cells.

\section{ACSL3 drives prostaglandin synthesis in NSCLC}

PI is the major source of AA, and in our lipidomic analysis, the AA-containing PI was consistently downregulated upon ACSL3 knockdown (Fig. 1a). Thus, we hypothesized that reduced AA-containing PIs would result in reduced prostaglandin synthesis. To this aim, we quantified PGE2, a prostaglandin produced by PGE2 synthase from COXderived prostaglandin $\mathrm{H} 2$ [24]. We found that PGE2 was strongly reduced upon ACSL3 knockdown in a panel of NSCLC cell lines harboring KRAS mutations, namely A549, A427, H1264, and H358, and this coincided with decreased cell proliferation (Fig. 1d, e and Supplementary Fig. 1C). To investigate whether ACSL3 knockdown impairs PGE2 production in lung cancer cells carrying wildtype KRAS, we assessed four representative cancer cell lines with known differential sensitivity to ACSL3 knockdown, namely H596, H838, H125, and HCC95 (ref. [17] and Supplementary Table 1). The proliferation of H596 and H838 cell lines was unaffected by ACSL3 knockdown (hereafter these cells are mentioned as ACSL3-independent), while the proliferation of H125 and HCC95 is significantly reduced (hereafter these cells are mentioned as ACSL3-dependent) (Supplementary Figs. 1D and 1E). We measured PGE2 upon ACSL3 knockdown with two different shRNAs, and found that the loss of ACSL3 caused either a mild decrease (H596) or had no effect (H838) in PGE2 production in the ACSL3-independent cancer cell lines, while it highly suppressed PGE2 production in the ACSL3-dependent cell lines H125 and HCC95 (Supplementary Fig. 1F).

To confirm these results in vivo, we generated a mouse model bearing a Cre-activatable mutant Kras allele ( $L S L$ $\left.\operatorname{Kras}^{G 12 D / W T}\right)$, homozygous for a Cre-conditional p53knockout allele $\left(p 53^{f l o x / f l o x}\right)[25,26]$ and either wild type $\left(L S L-K_{r a s}^{G 12 D / W T} ; p 53^{\text {flox/flox }} ; \mathrm{Acsl}^{+/+}\right)$or knockout for Acsl3 (LSL-Kras ${ }^{G 12 D / W T} ;$ p $^{\text {flox/flox }} ;$ Acsl3 $\left.^{-/}\right)$. In the LSL$\operatorname{Kras}^{G 12 D / W T} ; p 53^{\text {flox/flox }}$ model, Cre-mediated loss of a stop cassette permits expression of the oncogenic $\operatorname{Kras}^{G 12 D}$ allele from its endogenous promoter, and recapitulates key features of the human disease, including histologic features and response to conventional and targeted therapies [27]. Of note, we have previously shown that the $L S L-K_{r a s}{ }^{G 12 D / W T}$; $A c s l 3^{-/}$mice display impaired lung tumor initiation and progression compared with their wild-type littermates [17]. Therefore, we employed this mouse model to assess 
A

PC

PS

PE

PI

$\square$ pLKO

m shACSL3

$\square$ pLKO

ShACSL3
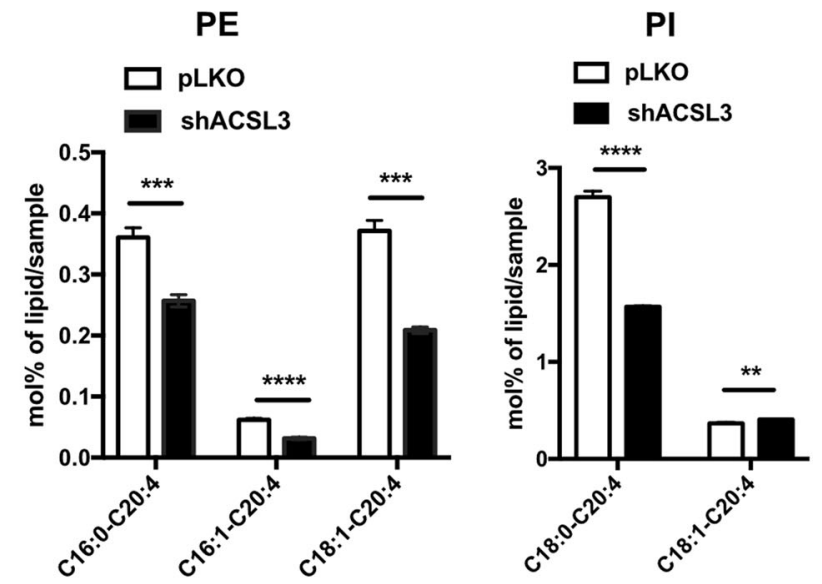

B
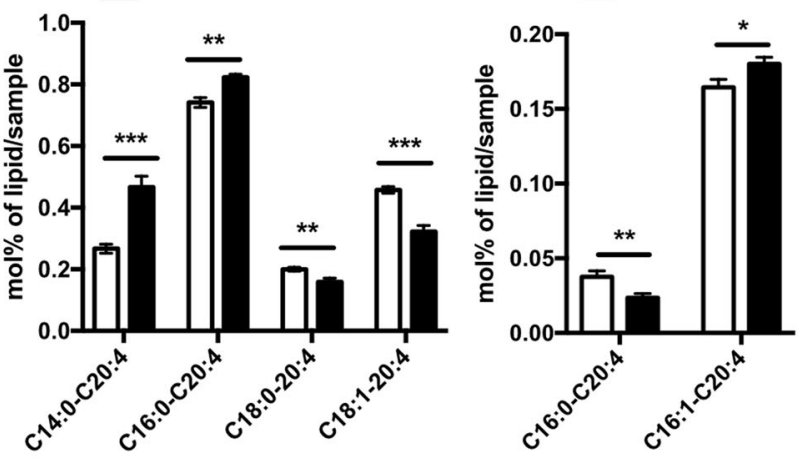

D

C
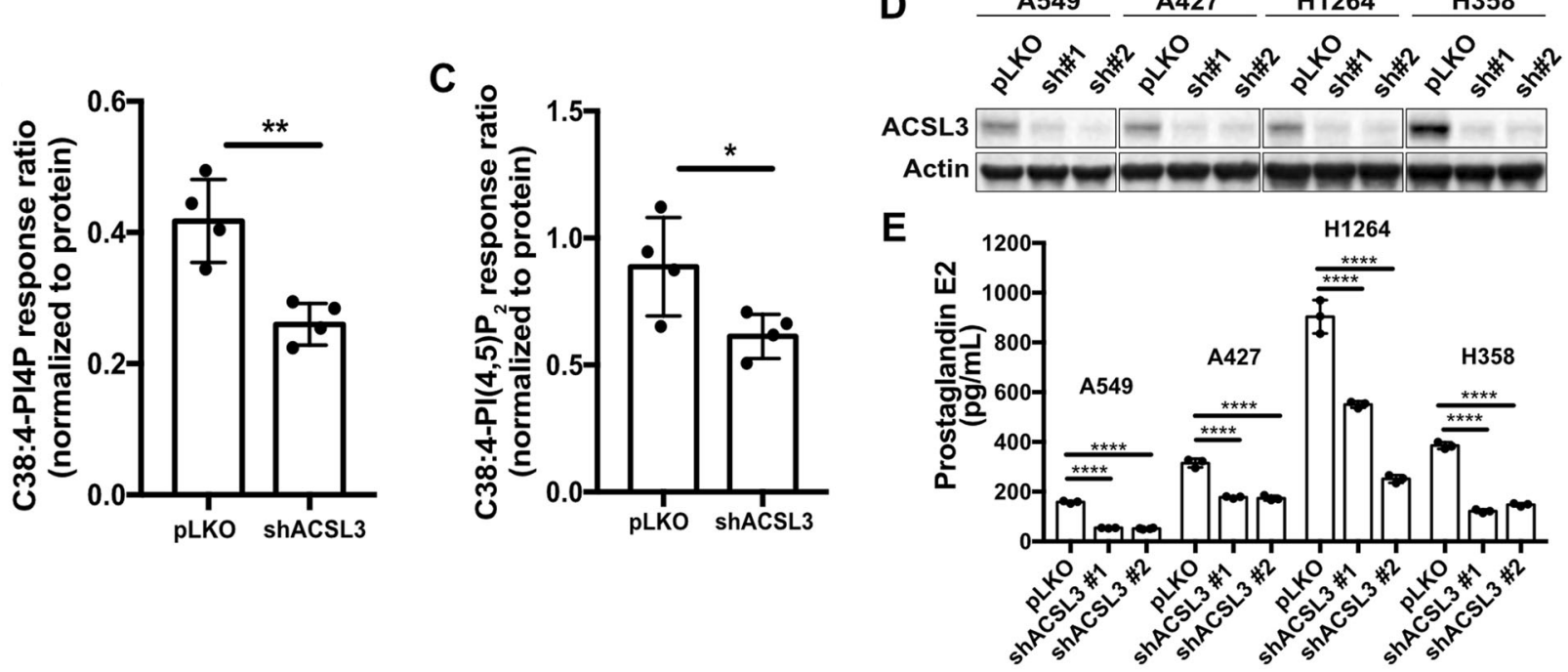

$\mathbf{F}$
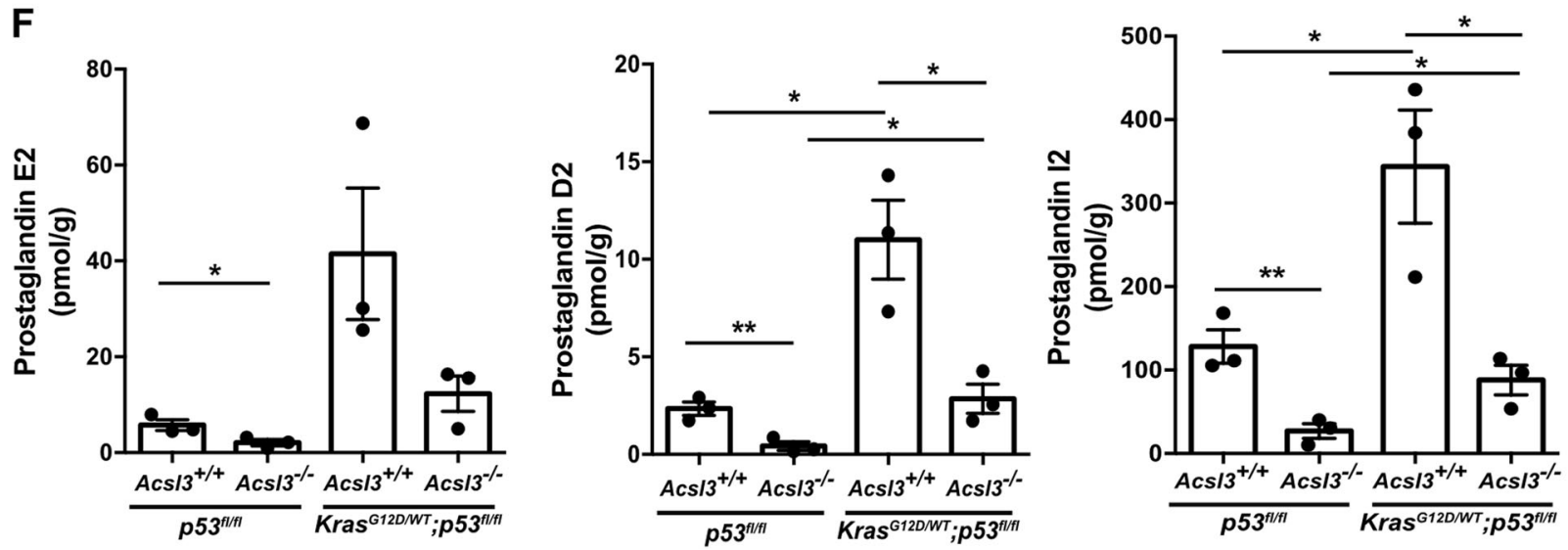

prostaglandin production in mouse lungs 10 weeks after adenoviral-mediated Cre delivery to the lungs. In both genotypes, the level of different prostaglandins (PGE2, PGD2, and PGI2) was significantly increased in tumor lesions compared with healthy lungs (Fig. 1f). However, we found in both lung tumors and healthy lungs that Acsl3 knockout causes a striking reduction of the aforementioned prostaglandins (Fig. 1f). Thus, our results suggest that ACSL3 is important for prostaglandin synthesis regardless of the oncogenic mutational status. Nevertheless, our data indicate that ACSL3 significantly enhances prostaglandin synthesis in tumors compared with healthy lung, suggesting that this may fulfill the increased requirement for prostaglandin synthesis of lung cancer. 
Fig. 1 ACSL3 drives prostaglandin synthesis in NSCLC. a PC, PS, $\mathrm{PE}$, and PI (mol\%/sample) lipid alterations $72 \mathrm{~h}$ after ACSL3 knockdown in A549 cells. Cells were transduced with either an empty vector control (pLKO) or an shRNA against ACSL3 (shACSL3 \#1), and $72 \mathrm{~h}$ later lipids were extracted and analyzed by mass spectrometry-based shotgun lipidomics. PC phosphatidylcholine, PS phosphatidylserine, PE phosphatidylethanolamine, PI phosphatidylinositol. $n=4$ /group. Data are presented as mean \pm SD. C38:4-PI4P (b), C38:4-PI $(4,5) \mathrm{P}_{2}$ (c) peak areas (normalized to internal standard and protein content) after ACSL3 knockdown in A549 cells. Cells were transduced with either an empty vector control (pLKO) or an shRNA against ACSL3 (shACSL3 \#1), and $72 \mathrm{~h}$ later lipids were extracted and analyzed by ultra-performance liquid chromatography-tandem mass spectrometry. $n=4$ /group. Data are presented as mean \pm SD. d Immunoblot analysis of ACSL3 in A549 and A427, H1264, and H358 NSCLC cell lines transduced with either an empty vector control (pLKO) or two different shRNAs against ACSL3 and extracted $72 \mathrm{~h}$ later. e PGE2 ELISA assay for the indicated cell lines. Cells were transduced with either an empty vector control (pLKO) or two different shRNAs against ACSL3. PGE2 production was analyzed $24 \mathrm{~h}$ later. $n=3$ / group. Data are presented as mean $\pm \mathrm{SD}$. f Quantification of prostaglandin E2, D2, and I2 from $p 53^{\text {floxfflox }} ; \mathrm{Acsl}^{+/+}$and $\mathrm{p} 53^{\text {flox } f \text { flox }}$;

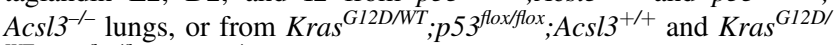
${ }^{W T} ; p 53^{\text {floxflox }} ; A c s l 3^{-/-}$macrodissected tumors, 10 weeks after tumor onset. Samples were extracted and analyzed by ultra-high-performance liquid chromatography with tandem mass spectrometry $n=3$ /group. Data are presented as mean \pm SEM. Statistical analyses were done using two-tailed unpaired Student's $t$ test or one-way ANOVA. ${ }^{*} p<$ $0.05, * * p<0.01, * * * p<0.001, * * * * p<0.0001$.

\section{LPIAT1 mediates prostaglandin production and promotes the proliferation and anchorage- independent growth of NSCLC cells}

Glycerophospholipids are remodeled by the action of phospholipases that hydrolyze them at the $s n-2$ position to yield a lysophospholipid and a free fatty acid, while their reacylation is catalyzed by lysophospholipid acyltransferases [28, 29]. Data from our lipidome profiling show that ACSL3 knockdown in A549 cells led to a reduction in C18:0-C20:4 PI, which could be caused by a decrease in C18:0-lysophosphatidylinositol (LPI) to C18:0-C20:4 PI production (Fig. 1a). Indeed, we found an accumulation of C18:0-LPI, suggesting that ACSL3 knockdown causes a block of LPI-PI conversion by reducing the supply of arachidonoyl-CoA (Fig. 2a).

The enzyme responsible for the conversion of LPI-PIs with specificity for arachidonyl-CoA is the lysophosphatidylinositolacyltransferase-1 (LPIAT1) (Fig. 2b) [30]. Thus, we hypothesized that LPIAT1 may be an important mediator of prostaglandin signaling downstream of ACSL3. Indeed, RNA interference-mediated LPIAT1 knockdown led to a significant decrease in PGE2 production in the mutant KRAS NSCLC cell lines, A549, A427, H1264, and H358 (Fig. 2c and Supplementary Fig. 2A). Next, we investigated whether LPIAT1 knockdown suppresses PGE2 production in wild-type KRAS NSCLC cell lines. LPIAT1 knockdown caused either a mild decrease (H596) or had no effect (H838) in PGE2 production in ACSL3-independent cancer cell lines, while significantly decreased PGE2 production in the ACSL3-dependent cell lines, H125 and HCC95 (Supplementary Figs. 2B and 2C). Moreover, in order to understand the impact of LPIAT1 depletion on cancer cell proliferation, we knocked down LPIAT1 in the aforementioned NSCLC cell lines. We found that LPIAT1 significantly suppressed the proliferation of mutant KRAS NSCLC cell lines A549, A427, H1264, and H358 (Fig. 2d) as well as that of ACSL3-dependent wild-type KRAS NSCLC cell lines, H125 and HCC95 but had no impact on the proliferation of H596 and H838 cell lines (Supplementary Fig. 2D). These results suggest that the ACSL3/LPIAT1 metabolic signaling axis plays an important role in prostaglandin production and cell proliferation of NSCLC cells.

To assess whether LPIAT1 requires ACSL3-derived substrates to control cancer cell proliferation, we overexpressed LPIAT1 with a lentiviral construct in A549 and H358 cell lines and performed cell proliferation assays upon ACSL3 knockdown. Interestingly, although the overexpression of LPIAT1 enhanced cancer cell proliferation, this effect was completely abolished upon ACSL3 knockdown, thus strongly suggesting that LPIAT1 requires ACSL3-derived arachidonyl-CoA to promote cancer cell proliferation (Fig. 2e and Supplementary Fig. 2E, 2F). Accordingly, measurement of PGE2 in A549 cells evidenced that in the absence of ACSL3, PGE2 production is compromised even when LPIAT1 is overexpressed (Fig. 2f). Next, we performed soft agar colony formation assays and we found that LPIAT1 overexpression increased the number and size of colonies, while the combination with ACSL3 knockdown abolished this effect (Fig. 2g, h). These data suggest that LPIAT1 requires ACSL3 to control cell proliferation and anchorage-independent growth in NSCLC cells.

\section{LPIAT1 knockdown suppresses tumorigenesis and improves the survival of mice bearing human lung cancer xenografts}

Our results suggest that LPIAT1 may be essential for KRAS-mediated prostaglandin production in lung cancer cells. In order to expand the significance of our finding in vivo, we injected the A549 (mutant KRAS) and HCC95 (wild-type KRAS, ACSL3-dependent) NSCLC cells into immunocompromised NOD scid gamma (NSG) mice, previously transduced with a control or shRNA against LPIAT1. In this model, we found that knockdown of LPIAT1 significantly suppressed tumorigenesis in mice and improved their overall survival (Fig. 3a-d). These results indicate that LPIAT1 plays a role in tumor progression. 
A

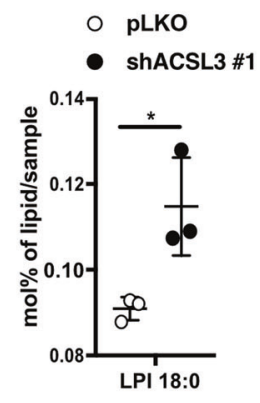

C

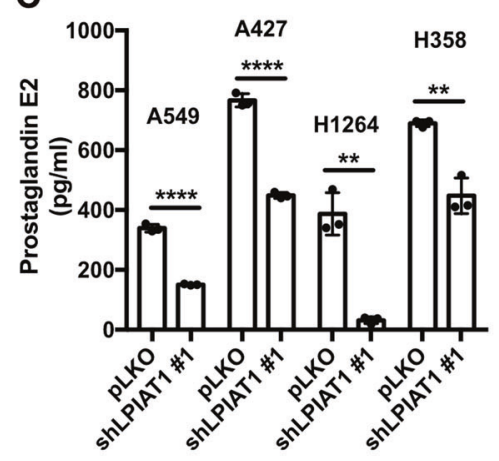

E Control

$\rightarrow$ shACSL3

$\vartheta$ LPIAT1 o/e

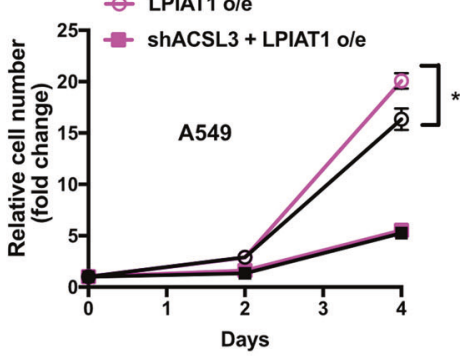

H $\square$ Control $\square$ LPIAT1 o/e

$\square$ shACSL3

shACSL3 + LPIAT1 o/e

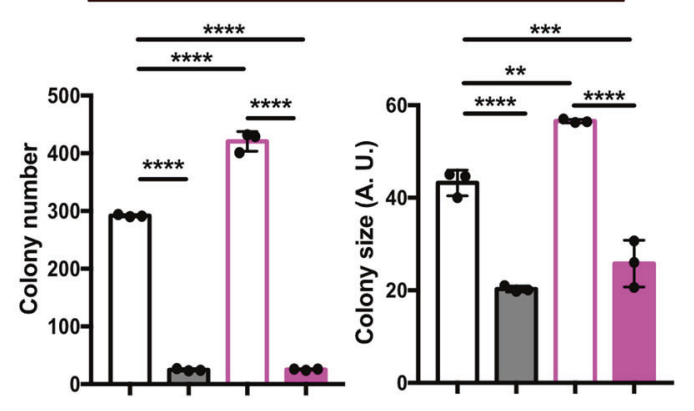

B

D

F
Free fatty acids

Acyl-CoA synthetases

Fatty acyl-CoAs CoA

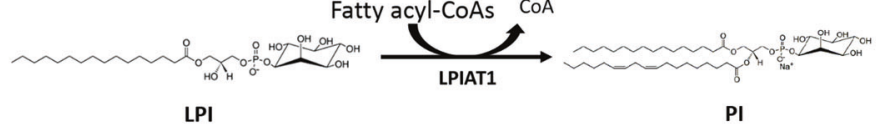

A549 (mut KRAS)

A427 (mut KRAS)

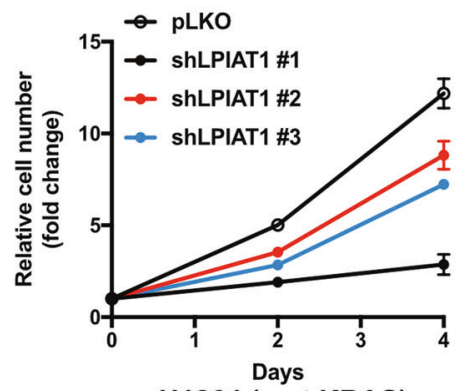

H1264 (mut KRAS)
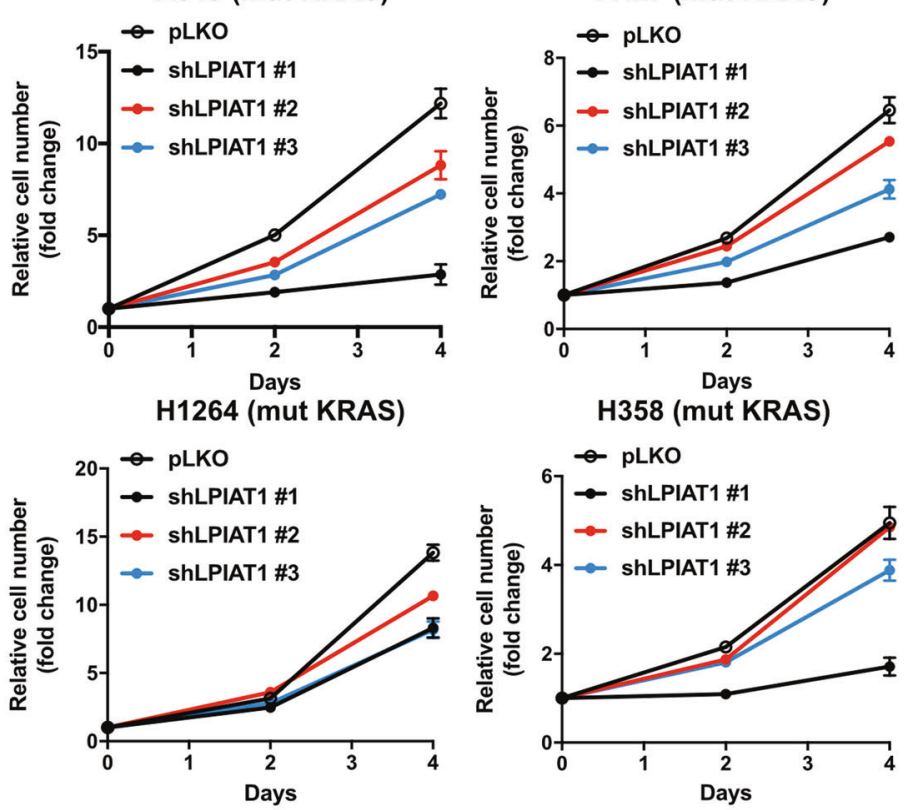

H358 (mut KRAS)

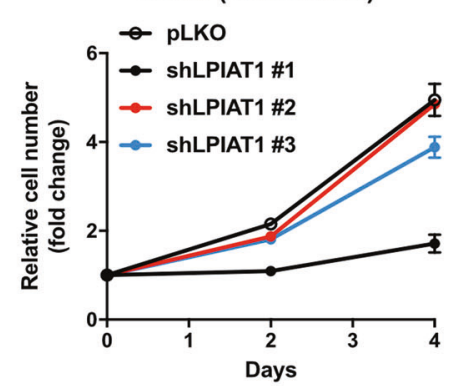<smiles>[134IH]</smiles>

G
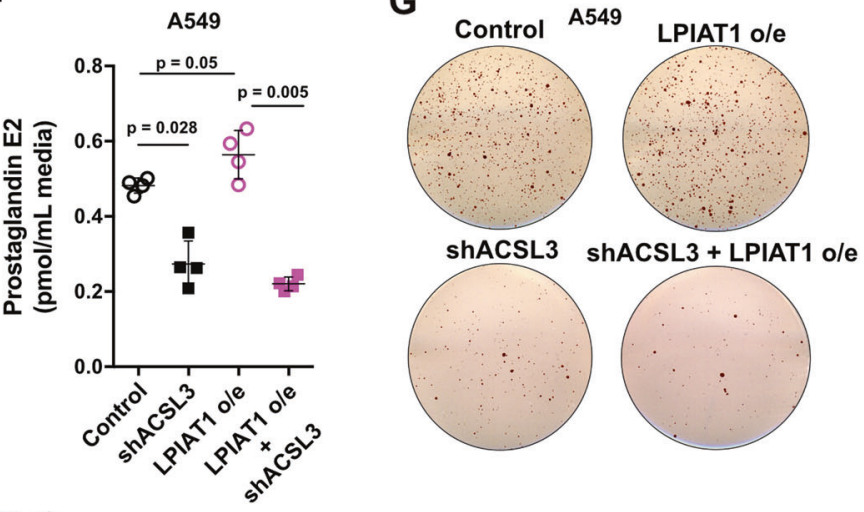
Fig. 2 LPIAT1 requires ACSL3-derived arachidonoyl-CoA for prostaglandin synthesis. a Lysophosphatidylinositol (LPI) $72 \mathrm{~h}$ after ACSL3 knockdown in A549 cells. Cells were transduced with either an empty vector control (pLKO) or an shRNA against ACSL3 (\#1), $72 \mathrm{~h}$ later lipids were extracted and analyzed by mass spectrometrybased shotgun lipidomics $n=4$ /group. Data are presented as mean \pm SD. b Schematic model for LPIAT1 function in phospholipid remodeling. c PGE2 ELISA assay for A549, A427, H1264, and H358 cell lines. Cells were transduced with either an empty vector control (pLKO) or shRNA against LPIAT1 (shLPIAT1 \#1). Cells were transduced, selected with puromycin and plated for PGE2 measurement. $24 \mathrm{~h}$ later the media was changed, incubated for additional $24 \mathrm{~h}$ and PGE2 production was quantified from the supernatant $n=3$ / group. Data are presented as mean \pm SD. d Relative cell number of A549, A427, H1264, and H358 cell lines transduced with an empty vector control (pLKO) or three different shRNAs against LPIAT1. $n=3$ /group. Data are presented as mean $\pm \mathrm{SD}$. e Relative cell number of A549 cell line transduced with either pLKO.1 hygro + pLenti-GIIICMV-GFP-2A-Puro (control) or pLKO.1 hygro-shACSL3 \#2+ pLenti-GIII-CMV-GFP-2A-Puro (shACSL3) or pLKO.1 hygro + pLenti-GIII-CMV-GFP-2A-Puro-LPIAT1 (LPIAT1 overexpression) or pLKO.1 hygro-shACSL3 \#2+ pLenti-GIII-CMV-GFP-2A-PuroLPIAT1 (shACSL3 + LPIAT1 overexpression) $n=3$ /group; o/e: overexpression. Data are presented as mean \pm SD. f PGE2 quantification from A549 cell line supernatants. Cells were transduced with either pLKO.1 hygro + pLenti-GIII-CMV-GFP-2A-Puro (control) or pLKO.1 hygro-shACSL3 \#2+ pLenti-GIII-CMV-GFP-2A-Puro (shACSL3) or pLKO.1 hygro + pLenti-GIII-CMV-GFP-2A-PuroLPIAT1 (LPIAT1 overexpression) or pLKO.1 hygro-shACSL3 \#2+ pLenti-GIII-CMV-GFP-2A-Puro-LPIAT1

(shACSL3 + LPIAT1 overexpression). Cells were transduced, selected with $2 \mu \mathrm{g} / \mathrm{mL}$ puromycin or $150 \mathrm{ng} / \mathrm{mL}$ hygromycin and plated for PGE2 measurement. $24 \mathrm{~h}$ later the media was changed, incubated for additional $24 \mathrm{~h}$ and Supernatants were analyzed by ultra-high performance liquid chromatography with tandem mass spectrometry $n=4$ /group. Data are presented as mean \pm SD. $\mathbf{g}$ Representative images of soft agar colony formation assay in A549 cells transduced as in f. h Histograms showing quantification of colony number and size of A549 cells grown in soft agar treated as in $\mathbf{f}$. $n=3$ /group. AU arbitrary units. Data are presented as mean \pm SD. Statistical analyses were done using twotailed unpaired Student's $t$ test or one-way ANOVA. $* p<0.05$, ${ }^{*} p<<$ $0.01, * * * * p<0.0001$

\section{LPIAT1 is upregulated in human NSCLC and positively correlates with poor patient survival}

To explore the relevance of LPIAT1 in lung cancer, we investigated a lung adenocarcinoma cohort (subset LUAD that includes information on KRAS mutational status) from the The Cancer Genome Atlas (TCGA) database, to compare the gene expression of LPIATl between wild-type KRAS tumors, mutant KRAS tumors and healthy lung tissue [31]. Our analysis evidenced a higher LPIAT1 expression in lung tumors compared with healthy lung tissue samples (Fig. 4a). However, the expression of LPIAT1 was higher in tumors with KRAS mutations compared with tumors carrying wild-type KRAS allele (Fig. 4a). Moreover, high LPIAT1 expression highly correlated with high Prostaglandin E Synthase expression, an enzyme that catalyzes the conversion of prostaglandin H2 to PGE2 (Fig. 4b). These data suggest that high LPIATl expression is not restricted to mutant KRAS tumors and underscore a broader relevance of LPIATI in NSCLC.

To validate the database analysis, we performed immunoblot using human patient-derived NSCLC biopsies with known KRAS mutations and the corresponding adjacent healthy tissue. Our results indicated that the protein levels of ACSL3, LPIAT1 and the cytosolic phospholipase A2 (cPLA2), an enzyme that catalyzes the hydrolysis of membrane phospholipids to release AA for prostaglandin and other eicosanoid production, are upregulated in patientderived mutant KRAS tumors compared with adjacent healthy tissue (Fig. 4c). Thus, these data confirm that the ACSL3-LPIAT1 metabolic pathway is enhanced in NSCLC.

To assess the relationship between ACSL3 and LPIATI expression, we employed a NSCLC cohort that includes squamous lung carcinomas (LUSC) and lung adenocarcinomas (LUAD), stratified by ACSL3-high and ACSL3-low expression, and we found a direct correlation between the expression levels of LPIATI and ACSL3, suggesting a coregulation of these enzymes in NSCLC (Fig. 4d).

Next, we examined the relationship between patient survival and ACSL3 or LPIAT1 expression. Kaplan-Meier analysis of LUSC and LUAD patient cohorts stratified by high versus low ACSL3 or LPIAT1, evidenced that patients with either high ACSL3 or high LPIAT1 expression had lower overall survival (Fig. 4e, f). These results suggest that both ACSL3 and LPIATI overexpression are clinically relevant and may have prognostic value for survival outcomes in NSCLC patients.

\section{Discussion}

Elevated prostaglandin levels have been extensively associated with enhancement of cancer cell survival and tumor growth, migration, invasion, and immunosuppression [3]. In several types of cancer, including mutant KRAS lung tumors, an important part of this effect has been attributed to the enhanced activity of COX1 and 2, the enzymes responsible for the production of prostaglandins from AA [32-34]. However, how the metabolism of AA is remodeled in cancer cells to cope with the high demand for prostaglandin synthesis remains elusive. Here, we found that, in mutant KRAS and in a subset of wild-type KRAS lung cancer cells, high prostaglandin levels are sustained by LPIAT1 activity and depend on the ACSL3-activated AA substrate availability (Fig. 2 and Supplementary Fig. 2). Importantly, the ACSL3-LPIAT1 metabolic axis drives prostaglandin synthesis to promote tumorigenesis in NSCLC (Fig. 3). We found that a subset of wild-type KRAS cancer cells show virtually no effect in PGE2 suppression and cell proliferation upon ACSL3 or LPIAT1 knockdown. These data suggest that alternative signaling pathways may confer resistance to ACSL3 or 
A

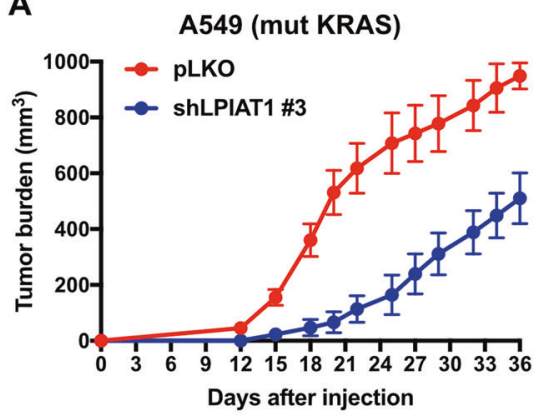

C

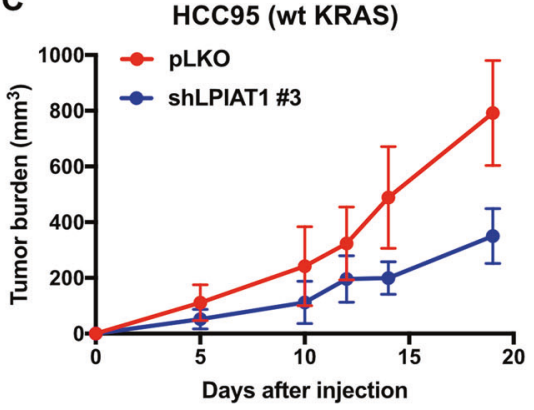

Fig. 3 LPIAT1 knockdown reduces tumorigenesis and extends survival of mice. a, c Tumor burden of A549 (mut KRAS) and HCC95 (wt KRAS) cells grown as xenografts in immunocompromised (NSG) mice. A549 or HCC95 cells were transduced with either a control (pLKO empty vector) or an shRNA against LPIAT1 (shLPIAT1 \#3) and $1 \times 10^{6}$ A549 or $2.5 \times 10^{6}$ HCC95 cells were injected subcutaneously in mice. Tumors were measured every

LPIAT1 inhibition and future studies will be necessary to identify these mechanisms. For instance, since the production of free AA is highly regulated by a PLA2-dependent deacylation reaction and a LPIAT1-dependent reacylation/transfer into PI pools, PLA2 overexpression may result in increased release of free AA available for prostaglandin synthesis leading to resistance to LPIAT1 inhibition. Indeed, cPLA2 overexpression is common in NSCLC [35]. Moreover, PLA2 enzymes can also liberate free AA from other phospholipids such as PC and PS [36]. Importantly, we found an upregulation of some PC and PS species bound to AA (C14:0-C20:4 PC, C16:0-C20:4 PC, and C16:1-C20:4 PS) upon ACSL3 knockdown, suggesting that ACSL3 suppression results in a remodeling of PC and PS membrane phospholipids that PLA2 could, in principle, deacylate to provide free AA for prostaglandin synthesis (Fig. 1a).

Our data show that cancer cell lines that are sensitive to ACSL3 knockdown are also sensitive to LPIAT1 knockdown, while others that are resistant to ACSL3 knockdown are also resistant to LPIAT1 knockdown (Supplementary Fig. 1D-1F and Supplementary Fig. 2B-2D). We also found that overexpression of LPIAT1 causes enhanced cancer cell proliferation and increased colony formation, an effect that was rescued by knocking down ACSL3 (Fig. 2e, h). In this context, knockdown of ACSL3 reduced the availability of
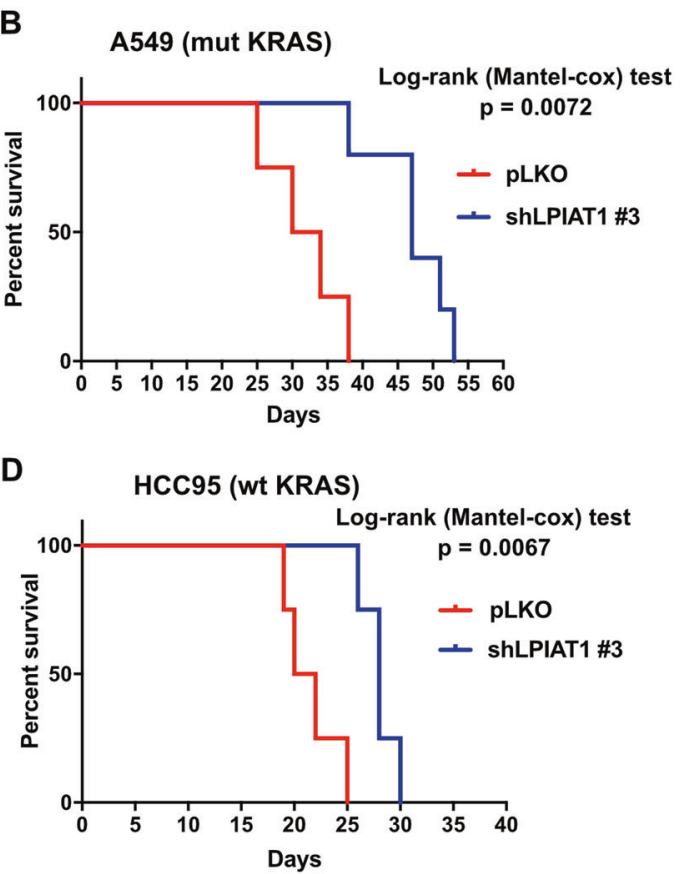

2-3 days with a caliper. Data in a are presented as mean \pm SEM and in $\mathbf{c}$ as \pm SD. b, d Kaplan-Meier survival curve of mice from A549 (b) or HCC95 (d) from the experiment shown in a and c, respectively. Statistical analyses were done using log-rank (Mantel-cox) test. A549 pLKO $n=4$, A549 shLPIAT1 $n=5$, HCC95 pLKO $n=4$, HCC95 $\operatorname{shLPIAT1~} n=4$.

arachidonoyl-CoA, the substrate of LPIAT1, hence reducing the most abundant PI species (C18:0-20:4 PI) and prostaglandin synthesis (Figs. 1a, d and 2f). Thus, our data indicate that ACSL3 and LPIAT1 regulate cancer cell proliferation, at least in part, by acting on the same metabolic axis. Whether the overexpression of ACSL3 is sufficient to increase synthesis of prostaglandins and promote cancer proliferation remains to be investigated.

We previously found that ACSL3 is required for fatty acid $\beta$-oxidation in NSCLC cells [17]. Therefore, it is plausible that LPIAT1 and ACSL3 can sustain lung cancer proliferation by also serving other pathways. In this regard, it has been recently shown that during human platelet activation, prostaglandins and other eicosanoids are fed at high rates into $\beta$-oxidation in a cPLA2-dependent manner. Accordingly, cPLA2 blockade decreased $\beta$-oxidation and impaired mitochondrial respiration [37]. Thus, it would be of interest to investigate whether lung cancer cells rely on prostaglandins as substrates to support $\beta$-oxidation.

Although nonsteroidal anti-inflammatory drugs, which target COX enzymes and specific COX2 inhibitors, are among the most promising drugs against cancer, the serious cardiovascular and gastrointestinal side effects have reduced the enthusiasm for their use [38]. Therefore, the identification of novel tumor-specific targets related to AA 
A

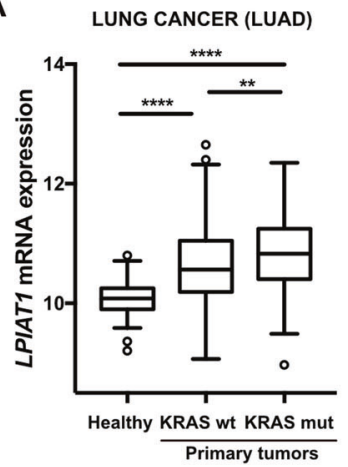

D LUNG CANCER (LUSC + LUAD)

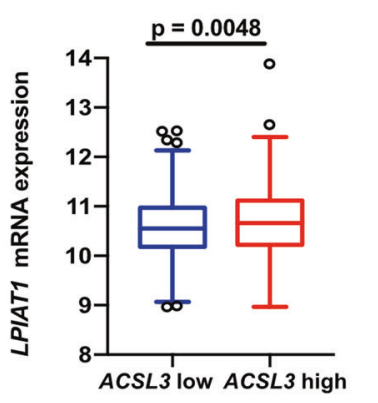

B

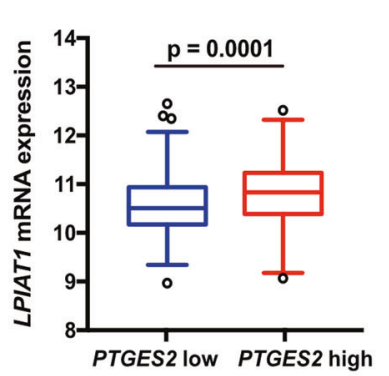

E

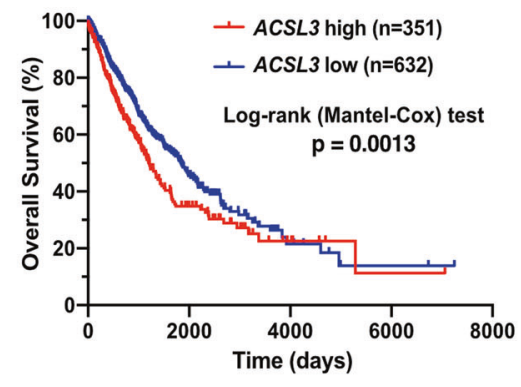

C

Patient \# 1

KRAS mutation - G12S $\overline{-G 12 C-G 12 C-G 12 D}$

$\begin{array}{llllllll}\mathbf{N} & \mathbf{T} & \mathbf{N} & \mathbf{T} & \mathrm{N} & \mathbf{T} & \mathbf{N} & \mathbf{T}\end{array}$

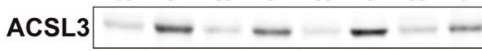

LPIAT1

cPLA2

$\beta$-actin $=-\infty-\infty-\infty-\infty$

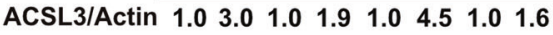

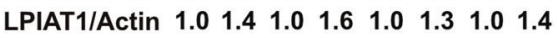

Fig. 4 LPIAT1 is overexpressed in human lung cancer and predicts poor patient survival. a Relative LPIAT1 mRNA expression in healthy lungs $(n=59)$ or lung tumors from wild-type $(n=326)$ or mutant KRAS $(n=148)$ human lung adenocarcinomas (LUAD cohort). The box-plots span from the first to third quartile (depicting the median as a line in the middle), the whiskers extend to $1.5 \mathrm{x} \mathrm{IQR}$ (interquartile range). Outliers $>1.5$ times the IQR are indicated with circles. b Relative LPIAT1 mRNA expression in LUAD cohort stratified by PTGES2-high $(n=258)$ versus PTGES2-low $(n=259)$ by median separation. Outliers $>1.5$ times the IQR are indicated with circles. c Immunoblot analysis of the indicated targets in primary human patient-derived lung adenocarcinoma samples. N normal lung,
T lung tumor (lung adenocarcinomas). The mutational status of KRAS is indicated. d Relative LPIAT1 mRNA expression in squamous lung carcinoma (LUSC) and lung adenocarcinoma (LUAD) cohorts stratified by ACSL3-high $(n=508)$ versus ACSL3-low $(n=509)$ by median separation. Outliers $>1.5$ times the IQR are indicated with circles. Kaplan-Meier analysis showing the percent survival of ACSL3-low versus ACSL3-high (e) or LPIAT1-low versus LPIAT1-high (f) in squamous lung carcinoma (LUSC) and lung adenocarcinoma (LUAD) cohorts. The number of patients is indicated. Statistical analyses were done using two-tailed unpaired Student's $t$ test, one-way ANOVA or log-rank (Mantel-cox) test. $* * p<0.01, * * * * p<0.0001$. remodeling may help develop therapies with a greater benefit and fewer side effects. Our results suggested that prostaglandin synthesis is higher in lung tumors compared with healthy tissue (Fig. 1f). Of note, mice with germline deletion of Acsl3 are viable and do not show any overt dysfunctions [17]. Thus, in principle, pharmacologic targeting of the ACSL3-LPIAT1 axis may benefit patients to selectively target tumor-derived prostaglandin synthesis while sparing normal cellular functions. However, germline deletion of LPIAT1 in mice results in defective brain development and mortality [39]. It would therefore be of interest to determine whether post-developmental knockout or inhibition of LPIAT1 would lead to a similar or other body dysfunctions, and whether it could be used for cancer treatment in the future. Notably, we found that high LPIATI expression predicts poor survival in mouse xenografts and human NSCLC patients (Figs. 3, 4). Hence, the status of ACSL3-LPIAT1 axis in human lung tumors may serve as a biomarker for personalized anti-cancer treatment.
Increased synthesis of prostaglandins is a negative prognostic marker in lung cancer and several other malignancies (e.g., gastric, colorectal, breast, hepatic, bladder, and renal cancers) $[10,40,41]$. Thus, future experiments aimed at assessing the role of LPIAT1 in prostaglandin synthesis and tumorigenesis in other types of cancer are warranted.

In conclusion, we have unraveled the ACSL3-LPIAT1 metabolic axis as a requirement for prostaglandin production and tumorigenesis in NSCLC that could be exploited for therapeutic intervention.

\section{Materials and methods}

\section{Resource sharing}

Further information and requests for resources and reagents should be directed to, and will be fulfilled by the Lead Contact, Georgia Konstantinidou, Institute of Pharmacology, 
University of Bern, 3010 Bern, Switzerland (georgia.konstantinidou@pki.unibe.ch). Requests will be handled according to the University of Bern policies regarding MTA and related matters.

\section{Reagents and plasmids}

pLKO.1 puro (Addgene plasmid \#8453; http://n2t.net/a ddgene:8453; RRID:Addgene_8453), pLKO.1 hygro (Addgene plasmid \#24150; http://n2t.net/addgene:24150; RRID:Addgene_24150), pCMV-VSV-G (Addgene plasmid \#8454; http://n2t.net/addgene:8454; RRID:Addgene_8454), and pCMV-dR8.2 dvpr (Addgene plasmid \#8455; http://n2t. net/addgene:8455; RRID:Addgene_8455) were a gift from Prof. Bob Weinberg [42]. The human LPIAT1-containing lentiviral vector (pLenti-GIII-CMV-GFP-2A-Puro) was purchased from Applied Biological Materials Inc. The ACSL3 and LPIAT1 shRNAs were obtained as bacterial glycerol stock from Sigma-Aldrich and the sequence of interest was subcloned into the pLKO-puro backbone or into pLKOhygro (for the combination studies with LPIAT1 overexpression) plasmids after digestion with AgeI/EcoRI. The final shRNA constructs were confirmed with sequencing.

\section{Cell lines}

All human NSCLC cell lines used in this study (A549, H358, H1264, A427, H838, H596, H125, and HCC95) were derived from male patients, and were provided by Dr. John Minna (UT Southwestern Medical Center) [43]. All cell lines were DNA fingerprinted for provenance. Cell lines were screened free for mycoplasma, and cultured in an incubator at $37{ }^{\circ} \mathrm{C}$ and $5 \% \mathrm{CO}_{2}$ in RPMI-1640 medium (Gibco) containing 10\% fetal bovine serum (Thermo Fisher), $100 \mathrm{I} . \mathrm{U} . / \mathrm{mL}$ penicillin, $100 \mu \mathrm{g} / \mathrm{mL}$ streptomycin, and $0.5 \mu \mathrm{g} / \mathrm{mL}$ puromycin (Gibco).

\section{Animal studies}

Mice were maintained under controlled humidity and temperature conditions, with a standard 12-h light/dark cycle and were fed ad libitum. Mixed background LSL-Kras ${ }^{G 12 D / N T}$; $p 53^{f l o x}$ fflox mice were generated by crossing stock B6.129SS4$\mathrm{kras}^{\text {tm } 4 T y j / J}$ (from JaxLab, Stock number 008179) [25], with B6.129P2-Trp53 $3^{\text {tmlBrn }} / J$ (from JaxLab, Stock number 008462) mouse [26]. The mixed background Cre-inducible LSL-Kras ${ }^{G I 2 D / W T} ; p 53^{\text {floxffox } ; A c s l 3^{-1-}}$ mouse model was

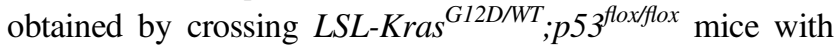
B6;129S5-Acs13 ${ }^{\text {Gt(OST148301)Lex } / O r l ~[44] . ~ L S L-K r a s ~}{ }^{G 12 D / W T}$; $p 53^{\text {floxffox}} ; \mathrm{Acsl}^{--}$mice were backcrossed for eight generations, before creating the experimental groups. The NOD.CgPrkdc $^{\text {scid }}$ Il2 rg $^{\text {tml } 1 \mathrm{Wjl} /} / \mathrm{SzJ}$ NSG mice were from Jackson labs (stock number: 005557). Only male littermates were used for the experiments. Animal handling and experimental procedures were performed in compliance with the federal guidelines and were approved by the Veterinaerdienst de Kantons Bern.

For intratracheal injections, $2.5 \times 10^{7}$ infectious particles of VVC-U of Iowa-5 Ad5CMVCre (Viral Vector Core, University of Iowa) were delivered to male mice at 8 weeks of age, resulting in the concomitant lung specific $\mathrm{Kras}^{\mathrm{Gl2D} /}$ WT induction, and p53 deletion. Mice were sacrificed 10 weeks post induction, and lungs were retrieved after anesthesia and perfusion of the animal with $20 \mathrm{ml}$ of PBS. Healthy lung tissue and macrodissected tumors used for measurement of prostaglandin levels were snap-frozen in liquid nitrogen.

For the xenotransplantation study in vivo, $1 \times 10^{6}$ A549 cells (A549 pLKO or A549 shLPIAT1 \#3) or $2.5 \times 10^{6}$ HCC95 cells (HCC95 pLKO or HCC95 shLPIAT1 \#3) resuspended in $100 \mu$ lof sterile PBS were injected subcutaneously to male NOD.Cg-Prkde ${ }^{\text {scid }} \mathrm{Il}_{2 \mathrm{rg}}{ }^{\text {tm } 1 \mathrm{Wj} /} / \mathrm{SzJ}$ NSG mice at 7-8 weeks of age. The mice were closely monitored on a daily basis, and the size of the tumors was measured with a caliper every 2-3 days. Mice were sacrificed when the tumor volume reached $1000 \mathrm{~mm}^{3}$.

Genomic DNA extraction and PCR assay were performed using the KAPA HotStart Mouse Genotyping Kit (Kapa Biosystems, KK7352) and KAPA2G Fast HotStart Genotyping Mix (Kapa Biosystems, KK5621), respectively, according to the manufacturer's instructions. The mice genotypes were confirmed following the corresponding provider's protocols. The full list of oligos used to genotype the mice can be found in Supplementary Table 2.

\section{Human studies}

The patient-derived frozen lung adenocarcinoma samples used for Fig. 4 were provided by the institute of pathology, translational research unit. The sex of the patients is the following: Patient \#1: female, \#2: female, \#3: male, and \#4: female. The use of human samples was approved by the ethics commission (swissethics), ID: 2017-01322. All samples were provided upon patients' consent.

\section{TCGA data analysis}

TCGA LUSC and lung adenocarcinoma (datasets LUSC and LUAD, respectively) datasets were retrieved from http://ca ncergenome.nih.gov. The data were downloaded with the help of the web graphic user interface Xenabrowser (xenabrowser.net) [45] and analyzed with GraphPad Prism version 7.0. Incomplete data, missing expression values and/ or survival were eliminated from the analysis and only primary tumors were considered. RSEM gene quantifications as provided by TCGA were taken. The stratification in high-/ 
low-expressing groups in Fig. 4b, d was performed by median separation as indicated in the related figure legends. For the patient survival analysis (Fig. 4e, f), patients were classified into two groups, and association between prognosis (survival) and gene expression (FPKM) was examined. The best expression cut-off refers the FPKM value that yields maximal difference with regards to survival between the two groups at the lowest log-rank $P$ value.

\section{shRNAs, virus production, and transduction}

Recombinant lentiviruses were produced by transfecting HEK 293 T cells, using the Trans $\mathrm{IT}^{\circ}-293$ Transfection Reagent (Mirus; MIR2705), with pCMV-VSV-G (VSV-G protein), pCMV-dR8.2 (lentivirus packaging vector), and lentiviral constructs, according to the manufacturer's instructions.

\section{Mass spectrometry-based shotgun lipidomics}

\section{Lipid extraction}

Mass spectrometry-based lipid analysis was performed by Lipotype GmbH (Dresden, Germany) as previously described [46]. Lipids were extracted using a two-step chloroform/ methanol procedure [47]. Data analysis and post-processing. Data were analyzed with in-house developed lipid identification software based on LipidXplorer [48, 49]. Data postprocessing and normalization were performed using an inhouse developed data management system. Only lipid identifications with a signal-to-noise ratio $>5$, and a signal intensity fivefold higher than that in the corresponding blank samples, were considered for further data analysis. Experimenters were blinded during data analysis.

\section{Ultra-performance liquid chromatography-tandem mass spectrometry}

\section{Reagents}

Methanol, chloroform, dichloromethane, and acetonitrile (Fisher) were all of mass spectrometry grade. Sodium formate and $\mathrm{HCl}$ were from Sigma, and TMS-diazomethane (TMS-DM, 2.0 M in hexanes) from Sigma-Aldrich and Acros. Lipid standards were ammonium salts of 1-heptadecanoyl-2-(5Z,8Z,11Z,14Z-eicosatetraenoyl)-sn-glycero-3phospho-(1'-myo-inositol-4', $5^{\prime}$-bisphosphate) [17:0-20:4 PI $\left.(4,5) \mathrm{P}_{2}\right]$ Avanti Polar Lipids, LM1904; 1-heptadecanoyl-2(5Z,8Z,11Z,14Z-eicosatetraenoyl)-sn-glycero-3-phospho-(1'myo-inositol-4'-phosphate) [17:0-20:4 PI(4)P] Avanti Polar Lipids, LM1901; and 1-heptadecanoyl-2-(5Z,8Z,11Z,14Zeicosatetraenoyl)-sn-glycero-3-phospho-( $1^{\prime}$-myo-inositol$3^{\prime}, 4^{\prime}, 5^{\prime}$-trisphosphate) [17:0-20:4 $\left.\mathrm{PI}(3,4,5) \mathrm{P}_{3}\right]$ Avanti Polar
Lipids, LM1906, Trimyristin (14:0, 14:0, 14:0), Tripalmitin (16:0, 16:0, 16:0), Triolein $(18: 1,18: 1,18: 1)$ Trilinolein (18:2,18:2,18:2), Tristearin (18:0,18:0,18:0), Triarachidin (20:0,20:0,20:0), Triarachidonin $(20: 4,20: 4,20: 4)$ (NuChekPrep, Inc. Elysian, MN).

\section{Sample processing}

Cells $\left(2 \times 10^{6}\right)$ were washed twice with PBS and incubated with $0.5 \mathrm{M}$ trichloroacetic acid (TCA) for $5 \mathrm{~min}$ on ice. Cells were then scraped from the dish, vortexed for $30 \mathrm{~s}$ and further incubated on ice for $5 \mathrm{~min}$. The TCA-treated samples were centrifuged at $20,000 \times g$ for $3 \mathrm{~min}$ at $4{ }^{\circ} \mathrm{C}$. After discarding the supernatant, the pellet was resuspended in $1 \mathrm{~mL}$ of $5 \%(\mathrm{w} / \mathrm{v}) \mathrm{TCA}+10 \mathrm{mM}$ EDTA and centrifuged at $20,000 \times g$ for $3 \mathrm{~min}$ at $4{ }^{\circ} \mathrm{C}$. After repeating the same step once, the pellet was used for the lipid extraction.

\section{Lipid extraction}

Prior to lipid extraction the following lipid analytical internal standards were added to the TCA precipitates: 17:0-20:4 PI(4,5) $\mathrm{P}_{2}, 17: 0-20: 4 \mathrm{PI}(3,4,5) \mathrm{P}_{3}, 17: 0-20: 4$ PIP. Lipids were extracted using a modification of an acidified chloroform-methanol extraction protocol [50, 51]. It was initiated by adding $670 \mu \mathrm{L}$ of chloroform:methanol:12 N $\mathrm{HCl}(40: 80: 1)$ to the TCA precipitate followed by vigorous vortexing for $5 \mathrm{~min}$ and incubation for $10 \mathrm{~min}$ at $4{ }^{\circ} \mathrm{C}$. Then $650 \mu \mathrm{L}$ of ice-cold chloroform was added and the samples were vortexed for another $2 \mathrm{~min}$ and allowed to sit for $5 \mathrm{~min}$ at $4{ }^{\circ} \mathrm{C}$ after which $300 \mu \mathrm{L}$ of ice cold $1 \mathrm{M} \mathrm{HCl}$ was added. The samples were vortexed for $2 \mathrm{~min}$, centrifuged at $10,000 \times g$ for $2 \mathrm{~min}$, and the lower phase was then collected in a fresh $2-\mathrm{mL}$ microcentrifuge tube. Ice cold theoretical lower phase $(900 \mu \mathrm{l})$ generated by combining chloroform:methanol:1.74 M HCl mixture (86:14:1,v/v/v) was added to the upper phase and the mixture was vortexed and centrifuged. The lower phase was then combined with the previously collected lower phase and dried under a stream of $\mathrm{N}_{2}$ and subsequently methylated as previously described [52].

\section{Mass spectroscopy}

LC/MS was carried out essentially as previously described [52]. Aliquots of sample resuspended in $20-100 \mu \mathrm{L}$ of $100 \%$ mass spectroscopy grade methanol were injected with a Waters Acquity FTN autosampler into the UPLC/MS. Chromatography over a Waters Acquity UPLC C4 column (Waters Acquity UPLC Protein BEH C4, $1.7 \mu \mathrm{m} 1.1 \times 100$; $300 \mathrm{~A})$ was carried out with an acetonitrile formic acid gradient monitored by a Waters XEVO TQ-S MS/MS in multiple reaction monitoring mode using electrospray and 
positive ion mode. The gradient was initiated with $10 \mathrm{mM}$ formic acid in water $/ 10 \mathrm{mM}$ formic acid in acetonitrile $(33: 67 \mathrm{v} / \mathrm{v})$, held for $1 \mathrm{~min}$, then increased to $15: 85, \mathrm{v} / \mathrm{v}$ in 9 min following injection, held at $85 \%$ for $1 \mathrm{~min}$, then increased to $100 \%$ for an additional $3 \mathrm{~min}$, and then reequilibrated to starting conditions for $3 \mathrm{~min}$.

\section{Quantification of PGE2, PGD2, and PGI2 by LC-MS/MS}

\section{Sample preparation}

Lung tissue samples were weighted while still frozen and transferred into $2 \mathrm{~mL}$ XXTuff reinforced microvials (Bio Spec Products Inc., OK, USA) with three chrome-steel beads (diameter, $2.3 \mathrm{~mm}$; Bio Spec Products Inc., OK, USA) and the corresponding volume of $0.1 \mathrm{M}$ formic acid to reach $100 \mathrm{mg} / \mathrm{mL}$. Samples were homogenized using a Mini-Beadbeater-24 (Bio Spec Products Inc., OK, USA) and the extraction of AA, PGE2, PGD2, and PGI2 (since PGI2 is not metabolically stable, this analyte was quantified using its stable hydrolysis product 6k-PGF1a) as previously described [53]. LC-MS/MS conditions. LC-MS/MS analysis was performed using our previously described protocol [53] with some changes. We used a Shimadzu UFLC coupled to a TripleQuad 5500 QTRAP mass spectrometer (AB Sciex, Canada). The LC column was a Reprosil-PUR C18 column $(3 \mu \mathrm{m}$ particle size; $2 \times$ $50 \mathrm{~mm}$; Dr. A. Maisch HPLC GmbH, Germany) maintained at $40{ }^{\circ} \mathrm{C}$ with a mobile phase flow rate of $0.3 \mathrm{~mL} /$ min. The mobile phase composition was a mixture of (A) $2 \mathrm{mM}$ ammonium acetate plus $0.1 \%$ formic acid and (B) acetonitrile plus $0.1 \%$ formic acid. A gradient elution was used, starting with $95 \%$ of phase A and linear increase of phase B reaching $40 \%$ at minute 3 ; then the linear increase rate was decreased to reach $65 \% \mathrm{~B}$ at minute 9 . Finally, to flush the column, phase B was increased by $95 \%$ at minute 10 and kept for 4 min with a subsequent reequilibration by decreasing phase $\mathrm{B}$ down to $5 \%$. The total analysis time was $17 \mathrm{~min}$. For quantification, an 11point calibration curve was analyzed, determining the slope, intercept, and regression coefficient, and analytes concentration in the samples was calculated applying the model previously described [53]. The values were normalized to total proteins. Protein quantification was done with a BCA kit.

\section{PGE2 ELISA assay}

PGE2 levels were measured in culture medium of A549, A427, H1264, H358, H596, H838, H125, and HCC95 human NSCLC cell lines using solid-phase sandwich enzyme-linked immunosorbent assay (ELISA). Briefly, for every cell line $3-3.5 \times 10^{5}$ cells were plated in six-well plates in a standard volume of culture medium. The supernatant was collected at $24 \mathrm{~h}$, and the assay was performed according to the manufacturer's protocol (Cayman Chemical, 514010). The assay had a range from 7.8 to $1000 \mathrm{pg} / \mathrm{ml}$ and its sensitivity was $\sim 15 \mathrm{pg} / \mathrm{ml}$. Proteins from the adherent cells were then extracted in a standard volume of RIPA buffer and the total protein content was used as normalization factor for PGE2.

\section{Immunoblotting}

Cells were lysed in RIPA buffer $(50 \mathrm{mM}$ Tris- $\mathrm{HCl} \mathrm{pH} 8.0$, $150 \mathrm{mM} \mathrm{NaCl}, 1.0 \% \mathrm{NP}-40,0.5 \%$ sodium deoxycholate, $0.1 \%$ SDS) containing complete EDTA-free protease inhibitors (Roche) and $1 \mathrm{mM}$ PMSF. Samples were resolved by SDS-PAGE in Bio-Rad blotting chamber, transferred to nitrocellulose membrane using a semi-dry chamber (Bio $\mathrm{Rad}$ ) and blocked in 5\% BSA. Membranes were then incubated overnight at $4{ }^{\circ} \mathrm{C}$ with primary antibody diluted in $5 \%$ BSA in TBS containing $0.1 \%$ Tween. Secondary fluorescenttagged antibodies were from Li-Cor biosciences, and development was done in Li-Cor fluorescence-chemiluminescence detector. All antibodies and their dilutions are listed in Supplementary Table 2.

\section{RT-PCR}

RNA was extracted using the RNAeasy kit (QIAGEN, 74104), and cDNA was synthesized with the RevertAid First Strand cDNA Synthesis Kit (Thermo Scientific, K1622). RT-PCR was performed in 96-well plates (TreffLab) with FastSybr green (Thermo Scientific, 4367659). The normalization was performed with the $\Delta \Delta \mathrm{CT}$ method. The full list of the oligonucleotides used can be found in Supplementary Table 2 .

\section{Cell proliferation assay}

Cells were plated at low confluency in 24-well plates (8000 cells/well for A549, 9000 cells/well for H358, H1264, and A427) and allowed to proliferate for 48 or $96 \mathrm{~h}$. Cell viability was measured by crystal violet (Sigma-Aldrich) staining ( $0.1 \%$ in $20 \%$ methanol) of adherent cells after 10 min fixation in $4 \%$ paraformaldehyde (Sigma-Aldrich). After washing twice and air-drying, stained cells were decolored with $5 \%$ acetic acid, and $\mathrm{OD}_{600}$ was measured with a spectrophotometer.

\section{Soft agar colony formation assay}

Cells $\left(3 \times 10^{5} /\right.$ well $)$ were seeded on semi-solid agar medium (bottom layer $0.6 \%$ and top layer $0.4 \%$ mixed with cells) in 
a six-well plate. After 14-21 days cells were formalin-fixed and stained with $0.005 \%$ Iodonitrotetrazolium. The colonies were counted using a microscope.

\section{Statistical analysis}

All data sets were organized and analyzed in Microsoft excel 2016 and GraphPad Prism version 7.0.0 (GraphPad Software, San Diego, CA, USA, www.graphpad.com). All data presented are expressed as mean \pm SEM or \pm SD of three or more biological replicates/group (except for the in vivo experiments and the human data analysis, the number is indicated in the related figure legends). The mass spectrometry-based lipidomics analyses and PGE2 measurements were repeated twice. The significance of the results was determined by employing two-tailed unpaired Student's $t$ test and one- or two-way ANOVA (Tukey's post test) when more than two groups were compared, and significance is indicated in the related figure legends. No outliers were found in any data set and no animals or data were excluded from statistical analysis.

Acknowledgements The authors wish to thank Prof. Hiroyuki Arai (University of Tokyo, Japan) for providing the LPIAT1 antibody [39]. The NSCLC cell lines were a kind gift from Prof. John D. Minna (University of Southwestern Medical Center at Dallas, Texas, USA). Human tissues were provided by the Tissue Bank Bern. We thank Dr. Alexis Traynor-Kaplan (University of Washington, Medicinal Chemistry Mass Spectrometry Center, Washington, USA) for help with MS analysis. This study was supported by the Swiss National Science Foundation (SNSF) professorship PP00P3_163929, Novartis Foundation for medical-biological research \#16C190, UniBern Forschungsstiftung (to GK), and SNSF research grant \#163359 (to JG).

Author contributions Experiments were conceived and designed by GK and MS, performed by MS, CP, MRS, and IR, JG and IR performed lipid measurement quantifications by LC-MS/MS, and MG and MRS analyzed the human data. GK supervised the study. GK and MS wrote the paper.

\section{Compliance with ethical standards}

Conflict of interest The authors declare that they have no conflict of interest.

Publisher's note Springer Nature remains neutral with regard to jurisdictional claims in published maps and institutional affiliations.

Open Access This article is licensed under a Creative Commons Attribution 4.0 International License, which permits use, sharing, adaptation, distribution and reproduction in any medium or format, as long as you give appropriate credit to the original author(s) and the source, provide a link to the Creative Commons license, and indicate if changes were made. The images or other third party material in this article are included in the article's Creative Commons license, unless indicated otherwise in a credit line to the material. If material is not included in the article's Creative Commons license and your intended use is not permitted by statutory regulation or exceeds the permitted use, you will need to obtain permission directly from the copyright holder. To view a copy of this license, visit http://creativecommons. org/licenses/by/4.0/.

\section{References}

1. Perez R, Matabosch X, Llebaria A, Balboa MA, Balsinde J. Blockade of arachidonic acid incorporation into phospholipids induces apoptosis in U937 promonocytic cells. J Lipid Res. 2006;47:484-91.

2. Surette ME, Fonteh AN, Bernatchez C, Chilton FH. Perturbations in the control of cellular arachidonic acid levels block cell growth and induce apoptosis in HL-60 cells. Carcinogenesis. 1999;20:757-63.

3. Wang D, Dubois RN. Eicosanoids and cancer. Nat Rev Cancer. 2010;10:181-93.

4. Balsinde J, Winstead MV, Dennis EA. Phospholipase A(2) regulation of arachidonic acid mobilization. FEBS Lett. 2002;531:2-6.

5. Harris RE. Cyclooxygenase-2 (cox-2) blockade in the chemoprevention of cancers of the colon, breast, prostate, and lung. Inflammopharmacology. 2009;17:55-67.

6. Poligone B, Baldwin AS. Positive and negative regulation of NFkappaB by COX-2: roles of different prostaglandins. J Biol Chem. 2001;276:38658-64.

7. Baratelli F, Lin Y, Zhu L, Yang SC, Heuze-Vourc'h N, Zeng G, et al. Prostaglandin E2 induces FOXP3 gene expression and T regulatory cell function in human $\mathrm{CD} 4+\mathrm{T}$ cells. J Immunol. 2005; 175:1483-90.

8. Huang M, Stolina M, Sharma S, Mao JT, Zhu L, Miller PW, et al. Non-small cell lung cancer cyclooxygenase-2-dependent regulation of cytokine balance in lymphocytes and macrophages: upregulation of interleukin 10 and down-regulation of interleukin 12 production. Cancer Res. 1998;58:1208-16.

9. Stolina M, Sharma S, Lin Y, Dohadwala M, Gardner B, Luo J, et al. Specific inhibition of cyclooxygenase 2 restores antitumor reactivity by altering the balance of IL-10 and IL-12 synthesis. J Immunol. 2000;164:361-70.

10. Wang D, Dubois RN. Prostaglandins and cancer. Gut. 2006;55:115-22.

11. Ferrer I, Zugazagoitia J, Herbertz S, John W, Paz-Ares L, SchmidBindert G. KRAS-Mutant non-small cell lung cancer: From biology to therapy. Lung Cancer. 2018;124:53-64.

12. Smakman N, Kranenburg O, Vogten JM, Bloemendaal AL, van Diest P, Borel Rinkes IH. Cyclooxygenase-2 is a target of KRASD12, which facilitates the outgrowth of murine C26 colorectal liver metastases. Clin Cancer Res. 2005;11:41-48.

13. Sheng H, Williams CS, Shao J, Liang P, DuBois RN, Beauchamp $\mathrm{RD}$. Induction of cyclooxygenase- 2 by activated Ha-ras oncogene in Rat-1 fibroblasts and the role of mitogen-activated protein kinase pathway. J Biol Chem. 1998;273:22120-7.

14. Xie W, Herschman HR. v-src induces prostaglandin synthase 2 gene expression by activation of the c-Jun $\mathrm{N}$-terminal kinase and the c-Jun transcription factor. J Biol Chem. 1995;270:27622-8.

15. Xie W, Herschman HR. Transcriptional regulation of prostaglandin synthase 2 gene expression by platelet-derived growth factor and serum. J Biol Chem. 1996;271:31742-8.

16. Kamphorst JJ, Cross JR, Fan J, de Stanchina E, Mathew R, White EP, et al. Hypoxic and Ras-transformed cells support growth by scavenging unsaturated fatty acids from lysophospholipids. Proc Natl Acad Sci USA. 2013;110:8882-7.

17. Padanad MS, Konstantinidou G, Venkateswaran N, Melegari M, Rindhe S, Mitsche M. et al. Fatty acid oxidation mediated by Acyl-CoA synthetase long chain 3 is required for mutant KRAS. Cell Rep. 2016;16:1614-28.

18. Mashek DG, Li LO, Coleman RA. Long-chain acyl-CoA synthetases and fatty acid channeling. Future Lipidol. 2007;2:465-76. 
19. Jungalwala FB, Evans JE, McCluer RH. Compositional and molecular species analysis of phospholipids by high performance liquid chromatography coupled with chemical ionization mass spectrometry. J Lipid Res. 1984;25:738-49.

20. Patton GM, Fasulo JM, Robins SJ. Separation of phospholipids and individual molecular species of phospholipids by highperformance liquid chromatography. J Lipid Res. 1982;23:190-6.

21. Tanaka T, Iwawaki D, Sakamoto M, Takai Y, Morishige J, Murakami K, et al. Mechanisms of accumulation of arachidonate in phosphatidylinositol in yellowtail. A comparative study of acylation systems of phospholipids in rat and the fish species Seriola quinqueradiata. Eur J Biochem. 2003;270:1466-73.

22. D'Souza K, Epand RM. Enrichment of phosphatidylinositols with specific acyl chains. Biochim Biophys Acta. 2014;1838:1501-8.

23. Wymann MP, Schneiter R. Lipid signalling in disease. Nat Rev Mol Cell Biol. 2008;9:162-76.

24. Park JY, Pillinger MH, Abramson SB. Prostaglandin E2 synthesis and secretion: the role of PGE2 synthases. Clin Immunol. 2006;119:229-40.

25. Jackson EL, Willis N, Mercer K, Bronson RT, Crowley D, Montoya R, et al. Analysis of lung tumor initiation and progression using conditional expression of oncogenic K-ras. Genes Dev. 2001;15:3243-8.

26. Marino S, Vooijs M, van Der Gulden H, Jonkers J, Berns A. Induction of medulloblastomas in p53-null mutant mice by somatic inactivation of $\mathrm{Rb}$ in the external granular layer cells of the cerebellum. Genes Dev. 2000;14:994-1004.

27. Oliver TG, Mercer KL, Sayles LC, Burke JR, Mendus D, Lovejoy $\mathrm{KS}$, et al. Chronic cisplatin treatment promotes enhanced damage repair and tumor progression in a mouse model of lung cancer. Genes Dev. 2010;24:837-52.

28. Holub BJ, Kuksis A. Differential distribution of orthophosphate$32 \mathrm{P}$ and glycerol- $14 \mathrm{C}$ among molecular species of phosphatidylinositols of rat liver in vivo. J Lipid Res. 1971;12:699-705.

29. Lands WE. Metabolism of glycerolipides; a comparison of lecithin and triglyceride synthesis. J Biol Chem. 1958;231:883-8.

30. Lee HC, Inoue $\mathrm{T}$, Imae R, Kono N, Shirae S, Matsuda $\mathrm{S}$, et al. Caenorhabditis elegans mboa-7, a member of the MBOAT family, is required for selective incorporation of polyunsaturated fatty acids into phosphatidylinositol. Mol Biol Cell. 2008;19:1174-84.

31. Cancer Genome Atlas Research Network. Author correction: comprehensive molecular profiling of lung adenocarcinoma. Nature. 2018;559:E12.

32. Dannenberg AJ, Subbaramaiah K. Targeting cyclooxygenase- 2 in human neoplasia: rationale and promise. Cancer Cell. 2003;4:431-6.

33. Howe LR, Subbaramaiah K, Patel J, Masferrer JL, Deora A, Hudis $\mathrm{C}$, et al. Celecoxib, a selective cyclooxygenase 2 inhibitor, protects against human epidermal growth factor receptor 2 (HER-2)/ neu-induced breast cancer. Cancer Res. 2002;62:5405-7.

34. Subbaramaiah K, Norton L, Gerald W, Dannenberg AJ. Cyclooxygenase-2 is overexpressed in HER-2/neu-positive breast cancer: evidence for involvement of AP-1 and PEA3. J Biol Chem. 2002;277:18649-57.

35. Blaine SA, Wick M, Dessev C, Nemenoff RA. Induction of cPLA2 in lung epithelial cells and non-small cell lung cancer is mediated by Sp1 and c-Jun. J Biol Chem. 2001;276:42737-43.

36. Quach ND, Arnold RD, Cummings BS. Secretory phospholipase A2 enzymes as pharmacological targets for treatment of disease. Biochem Pharm. 2014;90:338-48.
37. Slatter DA, Aldrovandi M, O'Connor A, Allen SM, Brasher CJ, Murphy RC, et al. Mapping the human platelet lipidome reveals cytosolic phospholipase A2 as a regulator of mitochondrial bioenergetics during activation. Cell Metab. 2016;23:930-44.

38. Borer JS, Simon LS. Cardiovascular and gastrointestinal effects of COX-2 inhibitors and NSAIDs: achieving a balance. Arthritis Res Ther. 2005;7Suppl 4:S14-22.

39. Lee HC, Inoue T, Sasaki J, Kubo T, Matsuda S, Nakasaki Y, et al. LPIAT1 regulates arachidonic acid content in phosphatidylinositol and is required for cortical lamination in mice. Mol Biol Cell. 2012;23:4689-4700.

40. Kaminska K, Szczylik C, Lian F, Czarnecka AM. The role of prostaglandin E2 in renal cell cancer development: future implications for prognosis and therapy. Future Oncol. 2014;10:2177-87.

41. Wang Z, Chen JQ, Liu JL. COX-2 inhibitors and gastric cancer. Gastroenterol Res Pract. 2014;2014:132320

42. Stewart SA, Dykxhoorn DM, Palliser D, Mizuno H, Yu EY, An DS, et al. Lentivirus-delivered stable gene silencing by RNAi in primary cells. RNA. 2003;9:493-501.

43. Phelps RM, Johnson BE, Ihde DC, Gazdar AF, Carbone DP, McClintock PR, et al. NCI-navy medical oncology branch cell line data base. J Cell Biochem Suppl. 1996;24:32-91.

44. Meehan TF, Chen CK, Koscielny G, Relac M, Wilkinson P, Flicek $\mathrm{P}$, et al. INFRAFRONTIER - providing mutant mouse resources as research tools for the international scientific community. Nucleic Acids Res. 2015;43:D1171-5.

45. Goldman M, Craft B, Kamath A, Brooks AN, Zhu J, Haussler D, The UCSC Xena platform for cancer genomics data visualization and interpretation. Preprint at https://www.biorxiv.org/content/10. 1101/326470v3 2018.

46. Sampaio JL, Gerl MJ, Klose C, Ejsing CS, Beug H, Simons K, et al. Membrane lipidome of an epithelial cell line. Proc Natl Acad Sci USA. 2011;108:1903-7.

47. Ejsing CS, Sampaio JL, Surendranath V, Duchoslav E, Ekroos K, Klemm RW, et al. Global analysis of the yeast lipidome by quantitative shotgun mass spectrometry. Proc Natl Acad Sci USA. 2009;106:2136-41.

48. Herzog R, Schwudke D, Schuhmann K, Sampaio JL, Bornstein SR, Schroeder M, et al. A novel informatics concept for highthroughput shotgun lipidomics based on the molecular fragmentation query language. Genome Biol. 2011;12:R8.

49. Herzog R, Schuhmann K, Schwudke D, Sampaio JL, Bornstein SR, Schroeder M. et al. LipidXplorer: a software for consensual cross-platform lipidomics. PLoS ONE. 2012;7:e29851

50. Traynor-Kaplan AE, Harris AL, Thompson BL, Taylor P, Sklar LA. An inositol tetrakisphosphate-containing phospholipid in activated neutrophils. Nature. 1988;334:353-6.

51. Traynor-Kaplan AE, Thompson BL, Harris AL, Taylor P, Omann GM, Sklar LA. Transient increase in phosphatidylinositol 3,4bisphosphate and phosphatidylinositol trisphosphate during activation of human neutrophils. J Biol Chem. 1989;264:15668-73.

52. Traynor-Kaplan A, Kruse M, Dickson EJ, Dai G, Vivas O, Yu H, et al. Fatty-acyl chain profiles of cellular phosphoinositides. Biochimica et biophysica acta Mol cell Biol lipids. 2017;1862:513-22.

53. Chicca A, Nicolussi S, Bartholomaus R, Blunder M, Aparisi Rey A, Petrucci V, et al. Chemical probes to potently and selectively inhibit endocannabinoid cellular reuptake. Proc Natl Acad Sci USA. 2017;114:E5006-15. 